\title{
Chronic stress effects on hippocampal structure and synaptic function: relevance for depression and normalization by anti-glucocorticoid treatment
}

\author{
Harmen J. Krugers' ${ }^{1}$, Paul J. Lucassen ${ }^{1}$, Henk Karst ${ }^{2}$ and Marian Joëls $s^{1,2 *}$ \\ 1 Center for Neuroscience, Swammerdam Institute for Life Sciences, University of Amsterdam, Amsterdam, Netherlands \\ 2 Department of Neuroscience and Pharmacology, Rudolf Magnus Institute of Neuroscience, University Medical Center Utrecht, Utrecht, Netherlands
}

\section{Edited by:}

Steven A. Kushner, Erasmus Medical Center, Netherlands

Reviewed by:

Monica DiLuca, University of Milano, Italy

Isabel Perez-Otano, University of Navarra Medical School, Spain; Centro de Investigacion en Medicina Aplicada, Spain

\section{${ }^{*}$ Correspondence:}

Marian Joëls, Department of Neuroscience and Pharmacology, Rudolf Magnus Institute of

Neuroscience, University Medical Center Utrecht, P.O. Box 85060, 3508

AB Utrecht, Netherlands.

e-mail:m.joels@umcutrecht.nl
Exposure of an organism to environmental challenges activates two hormonal systems that help the organism to adapt. As part of this adaptational process, brain processes are changed such that appropriate behavioral strategies are selected that allow optimal performance at the short term, while relevant information is stored for the future. Over the past years it has become evident that chronic uncontrollable and unpredictable stress also exerts profound effects on structure and function of limbic neurons, but the impact of chronic stress is not a mere accumulation of repeated episodes of acute stress exposure. Dendritic trees are reduced in some regions but expanded in others, and cells are generally exposed to a higher calcium load upon depolarization. Synaptic strengthening is largely impaired. Neurotransmitter responses are also changed, e.g., responses to serotonin. We here discuss: (a) the main cellular effects after chronic stress with emphasis on the hippocampus, (b) how such effects could contribute to the development of psychopathology in genetically vulnerable individuals, and (c) their normalization by brief treatment with anti-glucocorticoids.

Keywords: hippocampus, neurogenesis, LTP, chronic stress, depression

\section{INTRODUCTION}

Environmental challenges imposed on an individual are generally perceived and processed via the brain. This can lead to activation of parvocellular neurons in the hypothalamic paraventricular nucleus (see for reviews de Kloet et al., 2005; Ulrich-Lai and Herman, 2009), causing stimulation of the autonomic nervous system and the hypothalamo-pituitary-adrenal (HPA) axis. Consequently, enhanced levels of (nor) adrenaline and corticosteroid hormones (corticosterone in most rodents and cortisol in humans) are released into the circulation. These two hormonal systems are instrumental in producing and replenishing energy resources that enable the organism to cope with the immediate challenge, while at the same time they suppress functions for which there is no immediate need (McEwen, 2007).

The brain is an important target organ for corticosteroids (reviewed in de Kloet et al., 2005). Both the high-affinity mineralocorticoid receptor (MR) and the lower affinity glucocorticoid receptor (GR) are highly expressed in specific brain regions, including important limbic areas such as the CA1 hippocampus, dentate gyrus, and basolateral amygdala (BLA). In interaction with other stress hormones (e.g., noradrenaline and corticotrophin releasing hormone), corticosteroids alter neuronal activity in areas that play a key role in attention, vigilance, selection of the appropriate behavioral strategy, and in the storage of memories for future use (Joëls and Baram, 2009). This can be seen as the central contribution to the general stress response, overall resulting in successful behavioral adaptation.
The mechanism by which these behavioral effects of corticosteroid hormones are achieved has in part become evident from electrophysiological studies over the past two decades (reviewed in Joëls et al., 2007). In short, exposure to high levels of corticosteroids directly after stress quickly raises neuronal activity in hippocampal cells, on the one hand by promoting synaptic strengthening as demonstrated with paradigms inducing long-term potentiation (LTP; Wiegert et al., 2006), on the other hand by enhancing the frequency of mEPSCs and glutamate release probability (Karst et al., 2005; Olijslagers et al., 2008). The latter rapid, non-genomic actions require expression of MRs. At the same time, a slower, genemediated cascade is started via GR-dependent pathways, a cascade that will affect neuronal properties some hours later, i.e., by the time that the rapid effects have subsided. In CA1 neurons, these slow GR-dependent effects mostly attenuate information transfer - e.g., by enhancing cell firing frequency accommodation and hampering the induction of LTP (Joëls and de Kloet, 1989; Kerr et al., 1989; Kim and Diamond, 2002) - thus preserving earlier encoded information and normalizing activity to the pre-stress level. Yet, there seems to be a substantial regional specificity for GR-signaling pathways. For instance, dentate granule cells appear to be relatively resistant to slow GR-mediated signaling (Karst and Joëls, 2003; Van Gemert et al., 2009); principal neurons in the BLA can even display enhanced excitability some hours after stress (Duvarci and Paré, 2007; Liebmann et al., 2008). The delayed GR-mediated excitatory effects in the BLA may in part develop secondary to a rapidonset MR-dependent enhancement of glutamatergic transmission 
(Karst et al., personal communication). This would allow an extended time-window during which stress-related information can be encoded in the amygdala. If so, this might explain the observation that emotional or arousing details experienced in a stressful context are usually better remembered than neutral information (e.g., Buchanan and Lovallo, 2001; Smeets et al., 2009).

While activation of stress systems in response to a single challenge helps to restore homeostasis and is thus highly adaptive, repeated exposure to stressful situations - particularly when these are unpredictable, uncontrollable and/or taking place at vulnerable periods in life-can introduce a considerable risk for psychopathology, especially in genetically predisposed individuals. For instance, the chances to develop an episode of depression in people carrying two alleles of the serotonin transporter s-type gene variant increase with the number of stressful events they have experienced throughout life, whereas l-type gene variant carriers are relatively protected against depression under adverse life conditions (Caspi et al., 2003; but see Munafo et al., 2009). Similarly, carriers of the (gain of function) GR Bc/1 polymorphism are more likely to develop depression over the course of their life (reviewed by Maneschijn et al., 2009). Conversely, carriers of the GR loss-of-function ER22/23EK variant were reported to respond more quickly to antidepressants (Van Rossum et al., 2006).

There is ample evidence that cumulative stress exposure in life indeed forms a risk factor for the precipitation of depression; the functionality of the HPA-axis and glucocorticoids play a key role in this process (see for reviews de Kloet et al., 2005; Holsboer and Ising, 2010). For instance, high-risk proband of individuals with depression were found to exhibit hyper-reactivity of the HPA-axis prior to the onset of clinical symptoms. Also, many individuals with depression have elevated 24 -h corticosteroid levels and escape dexamethasone-induced suppression of the HPA axis; normalization of these parameters generally precedes the relief of depressive symptoms, and the degree of HPA-axis normalization in turn predicts the relapse probability. Finally, anti-glucocorticoids given (as adjunct to antidepressants) to patients with psychotic depression accelerate and increase chances on successful treatment (DeBattista and Belanoff, 2006), provided that the anti-glucocorticoids levels are very high (Blasey et al., 2009).

Given the documented role of the HPA-axis and glucocorticoids in the etiology of depression, one may wonder how overexposure to stress and corticosteroids changes the structure and function of brain cells in such a way that an individual becomes more vulnerable to depression. Is the impact of chronic stress a mere accumulation of what is seen after acute stress exposure? To get a better understanding of the mechanisms that may contribute to the development of depression one could examine how (prolonged) stress changes cellular structure and function in key areas involved in depression and how this correlates with depressive-like symptoms such as learned helplessness and anhedonia in rodent models (Henn and Vollmayr, 2005). In this review we will summarize the main effects observed after prolonged corticosteroid treatment or stress exposure on both the structure and function of neurons in the rodent hippocampus, an area of interest for the etiology of depression. The rodent chronic stress model allows indepth investigation of these cellular endpoints (which is presently not possible in humans) and is generally regarded to be relevant to the situation of accumulating stress exposure throughout life in humans. Next, we will discuss how these cellular changes could contribute to the development of mood disorders and how they can be relieved by brief treatment with anti-glucocorticoids.

\section{DEPRESSION, CHRONIC STRESS, AND STRUCTURAL PLASTICITY}

Recent studies have identified alterations in structural plasticity that may contribute to the etiology of depression (Castrén, 2005; Lucassen et al., 2010b). One of the most consistent findings is that hippocampal volume is reduced by approximately $10-15 \%$ in depression (reviewed in Sapolsky, 2000; Czeh and Lucassen, 2007). Several structural substrates can explain the hippocampal volume loss, ranging from glial changes, reduced neurogenesis, dendritic atrophy, or shifts in fluid balance. Massive cell loss, though, is not a likely explanation for the reductions in hippocampal volume since many of the stress-induced structural changes are transient and disappear after recovery (Pham et al., 2003; Heine et al., 2004a). The following paragraphs will address how prolonged stress affects structural plasticity. In particular we will address the effects of prolonged stress and exposure to corticosteroid hormones on neurogenesis - which is required for the behavioral effects of antidepressants in animal studies - and changes in dendritic complexity.

\section{CHRONIC STRESS AND ADULT HIPPOCAMPAL NEUROGENESIS}

Adult neurogenesis refers to the birth of new neurons in the adult brain that occurs in most vertebrate species including humans (Gould, 2007; Zhao et al., 2008). Similar to embryonic development, neurogenesis in the adult hippocampus involves a multi-step process starting with the proliferation of progenitor cells, followed by morphological and physiological maturation and finally functional integration into the hippocampal network. Although the exact functional role of the newborn granular neurons is unclear, numerous reports suggest that adult neurogenesis is involved in the acquisition of spatial learning, in pattern separation and in anxiety (Aimone et al., 2006; Dupret et al., 2008; Clelland et al., 2009; Garthe et al., 2009; Jessberger et al., 2009; Revest et al., 2009).

Stress is a potent inhibitor of adult neurogenesis; consistently, psychosocial and/or physical stressors all inhibit one or more phases of the neurogenetic process (Leuner et al., 2006; Oomen et al., 2007; Leuner and Gould, 2010). Both brief unpredictable stress exposure and chronic mild unpredictable stress potently suppress proliferation (see Figure 1). In addition, prolonged mild stress exposure inhibits the survival of newborn cells (Lucassen et al., 2010a). The origin of these stress-induced reductions in proliferation can be diverse, ranging from, e.g., apoptosis of progenitor cells to cell cycle arrest. Apoptosis (not only of newborn cells) is indeed affected by stress (Heine et al., 2004a). Thus, 1 day after brief unpredictable stress a reversible reduction in proliferation is paralleled by a reversible increase in the numbers of apoptotic cells (Heine et al., 2004a), suggesting enhanced turnover of granular cells. One day following chronic mild stress, both proliferation and apoptosis appear to be reduced in the granular cell layer (Heine et al., 2004a). Overall, these effects of prolonged stress are also reversible (Heine et al., 2004a). In parallel with these effects of prolonged stress exposure on apoptosis and proliferation, an increase of the cell cycle inhibitor p27Kip1 occurs in the dentate 

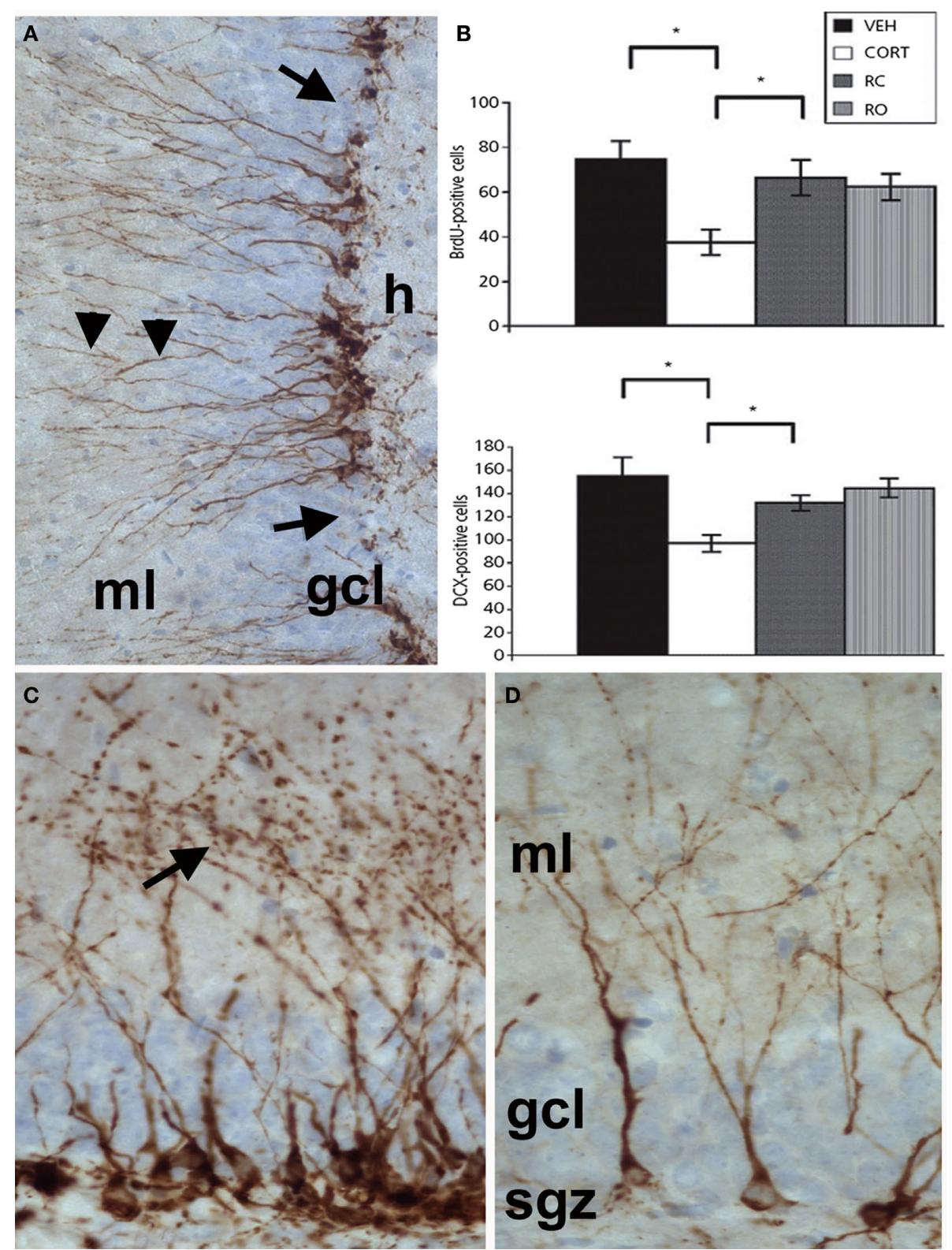

FIGURE 1 | (A) Doublecortin (DCX) immunostaining of immature neurons in the adult hippocampal dentate gyrus. DCX-positive somata are located in the subgranular zone at the border between the granular cell layer (gcl) and the hilus (h) with extensions (arrowheads) passing through the gcl and running into the molecular layer (ml). (B) Graph depicting quantification of BrdU- and DCXpositive cell numbers in the hippocampus of rats treated with vehicle (veh), corticosterone (cort) for 3 weeks or corticosterone for 3 weeks plus the GR-antagonist mifepristone (RC, applied during the last 4 days of corticosterone administration), and with mifepristone alone (RO, applied for 4 days). The significant reduction in both BrdU- (21-day old cells) and DCX-positive cell numbers after 21 days of corticosterone treatment is normalized by mifepristone treatment for the last 4 days, whereas the drug alone has no effect. Black bars: control animals; white bars animals treated with corticosterone for 3 weeks; dark gray bars: animals treated with corticosterone and RU 38486; light gray bars: animals treated with $R \cup 38486$. (C+D) Details of the individual morphological patterns of DCX-positive new neurons in the hippocampus of control (C) and stressed animals (D). Reproduced with permission from Mayer et al. (2006). gyrus (Heine et al., 2004b). The latter indicates that more cells enter cell cycle arrest, which may slow down granular cell turnover. However, the exact cellular pathways mediating the inhibitory effect of acute and prolonged mild stress on neurogenesis are unknown. Clearly, adrenal glucocorticoid hormones, via activation of GRs, appear to play a key role in this process (Montaron et al., 2003; Mayer et al., 2006; Boku et al., 2009 see Figure 1). At the same time, several examples exist of a persistent and lasting inhibition of adult neurogenesis after a brief stressor, despite the later normalization of glucocorticoid levels. These findings suggest that glucocorticoids may be involved in the initial suppression of cell proliferation, particularly in early life when adult neurogenesis is abundant, but that they are not always necessary for the maintenance of this effect. 
A large number of other factors may also mediate stress-induced inhibition of adult neurogenesis. For instance, NMDA receptors, which have been identified on early progenitor cells, may be involved (Nacher and McEwen, 2006) since blocking NMDA-receptors prevents the corticosterone-induced decrease in proliferating cells (Cameron et al., 1998). Stress is also known to affect the levels of various neurotransmitters such as GABA, serotonin, noradrenaline, dopamine, cannabinoids, opioids, nitric oxide, and several neuropeptides that have been reported to regulate adult neurogenesis (see Lucassen et al., 2010a). Furthermore, stress reduces the expression of a number of growth and neurotrophic factors that all can influence neurogenesis. Moreover, the proximity of the precursors to blood vessels suggests a strong interaction with the vasculature and it is this population that is particularly sensitive to prolonged mild stress (Heine et al., 2005). Also astrocytes, which support the survival of developing neurons, possess GRs and are significantly affected by prolonged psychosocial stress (Czéh et al., 2006).

Postmortem studies on the hippocampus of depressed patients have generally found reductions in proliferating cells (Reif et al., 2006; Lucassen et al., 2010b). Although reductions in neurogenesis may be characteristic for depression, it is unknown whether it is a cause, correlate, or consequence of the disorder (Kemperman and Kronenberg, 2003). In this respect it is of interest that a recent paper showed that reductions in neurogenesis can cause increases in stress hormone levels and HPA-axis activity rather than the other way around (Schloesser et al., 2009). In monkeys too, hippocampal volume appears predictive for stress responsivity (Lyons et al., 2007). Early life environment is also important, because in women with depression only those with a history of stress early in life exhibited a decreased hippocampal volume (Heim et al., 2008). Interestingly, perinatal stress in rodents affects critical periods of brain development and alters structural (including neurogenesis), emotional, and neuroendocrine parameters in adult offspring in a specific and persisting manner that depends very much on the type and timing of stress (Lemaire et al., 2000; Bredy et al., 2003; Fenoglio et al., 2006; Oomen et al., 2009, 2010). As such, a reduced rate in neurogenesis may be a vulnerability factor for depression.

\section{CHRONIC STRESS AND DENDRITIC COMPLEXITY}

In addition to the robust effects on neurogenesis, prolonged exposure to stress also dynamically regulates dendritic complexity. These stress-induced changes in dendritic structure are not only confined to the hippocampus but can also be observed in amygdala and prefrontal cortex. Moreover, stress can both increase and decrease dendritic complexity, depending on the brain area.

Stress-induced hippocampal structural plasticity has been reported in particular in the hippocampal CA3 area. Overall, prolonged exposure to glucocorticoids and prolonged psychosocial stress does not reduce cell number (Bodnoff et al., 1995; Vollmann-Honsdorf et al., 1997; Tata et al., 2006), but does cause a small reduction in total hippocampal CA3 volume (Czeh et al., 2001; Sheline et al., 2003; Heine et al., 2004a; Stewart et al., 2005). Moreover, there is evidence that glial processes make up a larger proportion of the tissue after exposure to prolonged psychosocial stress and glucocorticoid exposure (Czéh et al., 2006; Tata et al., 2006; Tata and Anderson, 2010). At the structural level, many studies have reported that prolonged exposure to glucocorticoids and stress reduces apical (but not basal) dendritic complexity and length of CA3 pyramidal neurons (Woolley et al., 1990; McEwen, 2000). Various types of chronic stress (repeated restraint stress, prolonged mild stress) resulted in similar changes in different species (Watanabe et al., 1992; Magarinos and McEwen, 1995; McKittrick et al., 1995, 2000; Magarinos et al., 1996; Galea et al., 1997). These changes in hippocampal dendritic morphology need several weeks to develop (Magarinos and McEwen, 1995), and the delay rather than the continuous presence of elevated corticosteroid levels seems to be important for these effects to occur (Magarinos and McEwen, 1995; Kole et al., 2004). Stressand glucocorticoid-induced remodeling of dendritic structure can be prevented by the anti-epileptic drug phenytoin implying that excitatory amino acids are critically involved in these effects (McEwen, 2000). Other neurotransmitters that have been implicated in stress-induced remodeling include serotonin and GABA (McEwen, 2000). Recent evidence also points to a critical role for tissue plasminogen activator (Pawlak et al., 2005) and brainderived neurotrophic factor (Magarinos et al., 2010).

In addition to changes in the apical dendritic tree, prolonged restraint stress or glucocorticoid exposure also leads to a loss of mossy fiber synapses (Sousa et al., 2000; Sandi et al., 2003; Tata et al., 2006). These changes at the synaptic level are accompanied by the retraction of thorny excrescences (Stewart et al., 2005). Presynaptically, prolonged stress/glucocorticoid exposure causes a rearrangement of synaptic mitochondria and vesicles, with more densely packed vesicle clusters localized close to the active zones (Magarinos et al., 1997). Taken together this indicates that prolonged exposure to chronic stress and glucocorticoids markedly change both pre-and post-synaptic structural structure.

The morphological changes in other hippocampal subfields upon exposure to prolonged chronic stress are less well studied. Prolonged activity-induced stress or corticosterone administration did show dendritic retraction of CA1 pyramidal neurons in some (Lambert et al., 1998; Sousa et al., 2000) but not in all studies (Woolley et al., 1990; Alfarez et al., 2008). Interestingly, brief exposure to elevated corticosteroid hormone levels does reduce apical CA1 dendritic complexity in animals that were exposed to prolonged mild stress (Alfarez et al., 2008; see Figure 2) indicating that prolonged stress renders these cells more sensitive to acute stress; this sensitivity to glucocorticoids was also observed in tissue from young animals (Alfarez et al., 2009). At the synaptic level, prolonged corticosteroid exposure decreases the number of spines on CA1 pyramidal neurons via a mechanism that requires tissue plasminogen activator (Pawlak et al., 2005).

Chronic stress not only affects hippocampal morphology. The prefrontal cortex and amygdala also appear to be remarkably sensitive to stress. Both brief and long-term exposure to restraint stress and glucocorticoid exposure elicit dendritic retraction of layer II/ III pyramidal neurons in the medial prefrontal cortex (mPFC) (Wellman, 2001; Seib and Wellman, 2003; Cook and Wellman, 2004; Brown et al., 2005; Izquierdo et al., 2006; Liston et al., 2006; Radley et al., 2006; Hains et al., 2009). Interestingly, there is dichotomy in the effects of stress on structural plasticity in the prefrontal cortex since dendritic complexity of pyramidal cells in the orbital frontal cortex - which play a role in reversal learning - is increased after prolonged stress (Liston et al., 2006). 


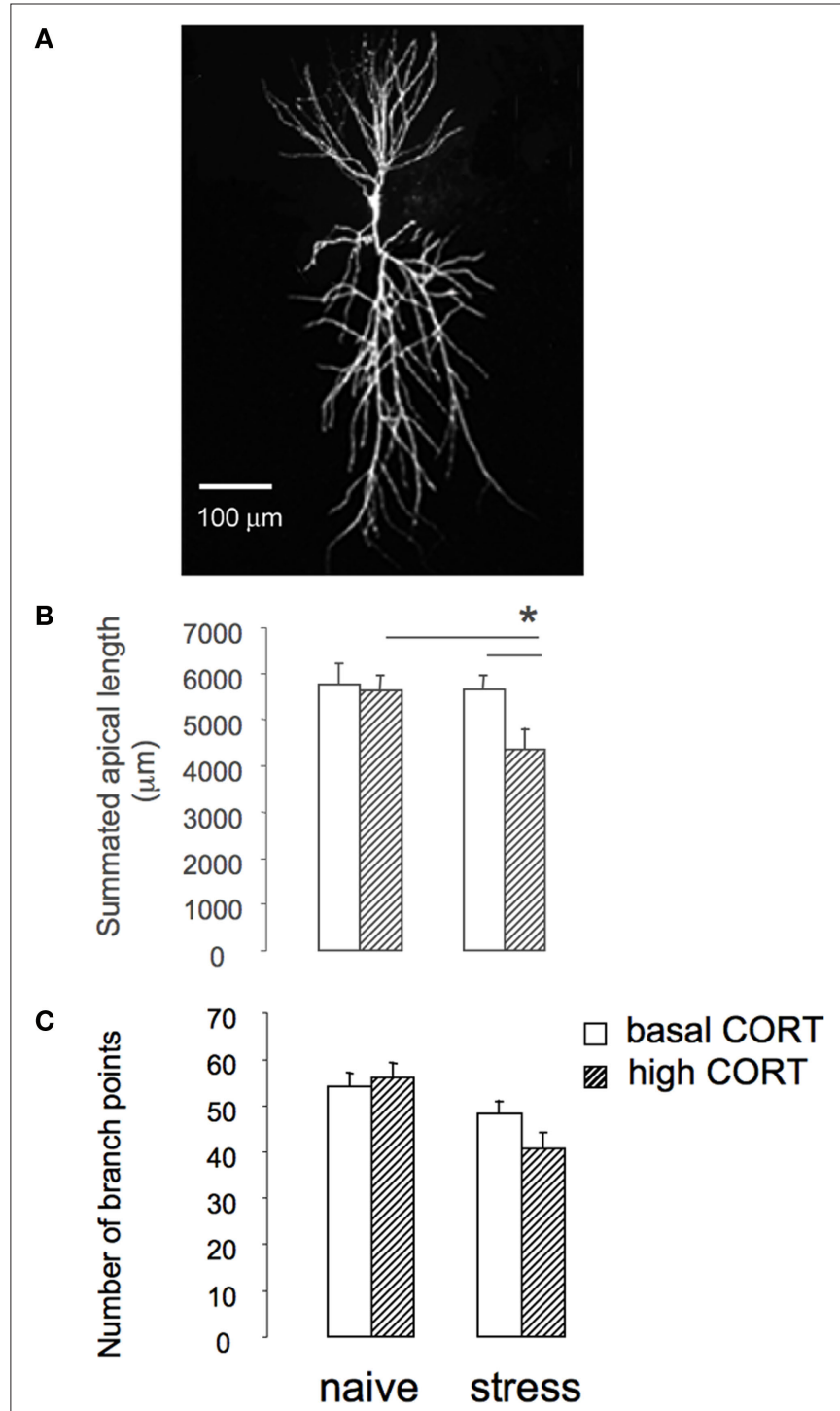

FIGURE 2 | Prolonged stress affects sensitivity of hippocampal CA1 structural plasticity. (A) Example of hippocampal CA1 pyramidal cell stained with Alexa 568. (B) Summated length of hippocampal CA1 pyramidal cells of naïve animals and animals exposed to 3 weeks of unpredictable stress. Exposure to prolonged stress does not affect length of CA1 pyramidal cells. However, brief application of corticosterone to stressed animals (dashed bars) rapidly reduces apical dendritic length. (C) Number of branch points of hippocampal CA1 pyramidal cells of naïve animals and animals exposed to 3 weeks of unpredictable stress (based on Alfarez et al., 2008).

The amygdala also appears to be extremely sensitive to prolonged stress. Exposure to chronic restraint stress triggers an increase in the total dendritic length as well as the number of spines of pyramidal and stellate cells in the BLA (Vyas et al., 2002, 2006). These effects may be stressor specific and/or cell specific since prolonged exposure to unpredictable stress reduces dendritic length of the bipolar cells (Vyas et al., 2002). Like in the CA3 region, stressinduced hypertrophy develops gradually in the amygdala and may be involved in the increased anxiety after (chronic) stress exposure (Mitra and Sapolsky, 2008).

\section{CHRONIC STRESS AND SYNAPTIC FUNCTION}

Several conspicuous changes in cell and/or network function have been observed after 3 weeks of exposure to high levels of corticosterone or stress. In all hippocampal areas, i.e., CA1, CA3, and dentate gyrus, induction of LTP is greatly impaired after a prolonged period of mild stress or chronic corticosteroid exposure (Bodnoff et al., 1995; Pavlides et al., 2002; Alfarez et al., 2003). This was seen both when corticosteroid levels are low (i.e., under conditions of rest, against a background of chronic stress), and several hours after a brief pulse of corticosterone (Alfarez et al., 2003). This indicates that activity-dependent strengthening of synapses, as is thought to occur in association with learning and memory formation, is greatly impaired after chronic stress under all circumstances.

The changes in synaptic strengthening may have been caused by alterations in glutamatergic transmission, the GABAergic network or calcium influx after chronic stress. In the CA3 area an enhancement of NMDA-receptor dependent transmission was observed (Kole et al., 2002) after prolonged psychosocial stress, while in the dentate gyrus AMPA-receptor dependent synaptic transmission mediated was found to be increased (Karst and Joëls, 2003), though only when chronic mild stress was combined with acute GR activation. In line with the generally raised basal excitability, a very recent report demonstrated reduced functionality of the GABAergic network in the hippocampal CA1 area after chronic restraint stress exposure in rats, presumably by a reduction in the number of parvalbumin-positive interneurons (Hu et al., 2010). The rapid increase in spontaneous GABAergic events seen in control animals after dexamethasone treatment was attenuated. These changes in basal glutamatergic and GABAergic transmission may implicate the potential to strengthen synaptic contacts.

Moreover, the combination of acute and chronic GR activation also enhanced calcium influx into dentate granule cells, compared to acute GR activation in handled controls (Van Gemert and Joëls, 2006). In CA1 pyramidal cells calcium influx was also increased, but this was most clearly seen in tissue from chronically stressed rats which were studied under rest (Karst and Joëls, 2007); after corticosterone application in vitro the amplitude declined (Figure 3). Enhanced calcium influx might hamper NMDA-receptor function and hence contribute to the generally impaired LTP observed after chronic stress.

Chronic stress also affects responsiveness of CA1 pyramidal neurons to serotonin (5-HT). In control animals, brief exposure of CA1 hippocampal cells to corticosterone induces a delayed GR-dependent enhancement of responses (i.e., a bigger hyperpolarization of the resting membrane potential) in the CA1 area via the 5-HT1A receptor (Karst et al., 2000). After 3 weeks of exposure to high levels of corticosterone or to unpredictable stress, 5-HT1A receptor-mediated responses were attenuated (Karten et al., 1999; Van Riel et al., 2003), both under basal corticosterone conditions and several hours after a brief exposure to corticosterone (i.e., when MRs as well as GRs are activated; Van Riel et al., 2003). The attenuated responses to serotonin after corticosterone treatment developed gradually and were associated with a significant reduction in the single cell expression level of MR relative to GR after 1 week of treatment, whereas expression levels of the 5-HT1A receptor itself were unchanged, both after 1 week and 3 weeks of corticosterone treatment (Karten et al., 1999). 


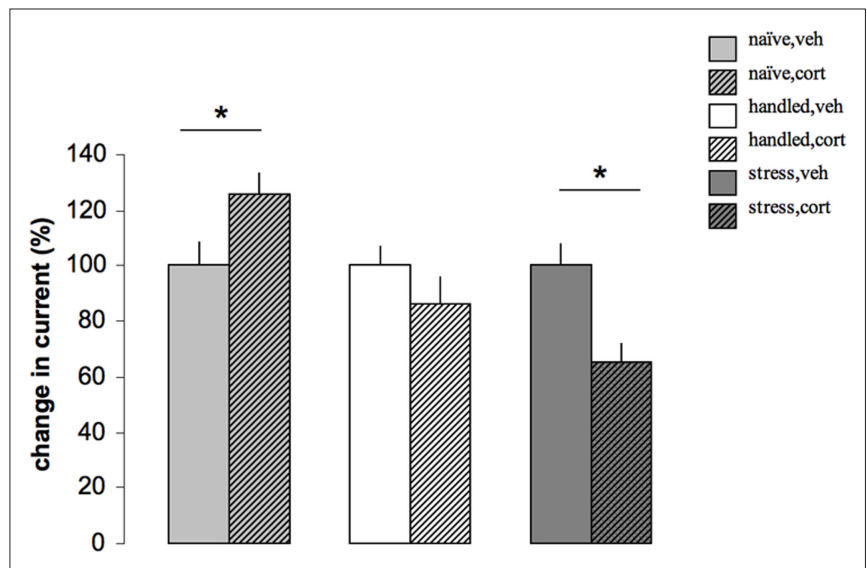

FIGURE 3 |The effect of corticosterone on calcium current amplitude depends on the stress history of the rat. Calcium currents were recorded with the whole cell voltage clamp method in CA1 pyramidal cells from adult male rats. For each cell, we constructed a current-voltage plot, inducing currents by successively depolarizing the membrane from a holding potential of $-70 \mathrm{mV}$, with an intermediate $3 \mathrm{~s}$ hyperpolarization to $-120 \mathrm{mV}$ to remove steady state inactivation; next, we determined the peak amplitude for the voltage step evoking the maximal current, which usually involved a step to $-20 \mathrm{mV}$. Part of the slices were pretreated for $20 \mathrm{~min}$. with $100 \mathrm{nM}$ corticosterone in vitro, 1-4 h before recording, to determine the responsiveness of CA1 neurons to this hormone. In naïve animals (left), acute corticosterone application (naïve, cort) led to a slow GR-dependent increase in calcium current amplitude compared to veh treated cells (naïve, veh; here arbitrarily set at $100 \%$, to allow comparison between the three experimental conditions). Animals that were handled for 3 weeks (middle), were relatively unresponsive to corticosterone (handled, cort) and showed amplitudes that were comparable to those seen after vehicle treatment (handled, veh). Yet, in animals that were exposed to 3 weeks of unpredictable stress (right), corticosterone reduced (stress, cort) rather than enhanced calcium current amplitude, compared to the amplitudes recorded several hours after vehicle treatment (stress, veh). For all conditions, calcium current amplitude after vehicle treatment was normalized to $100 \%$ (based on Karst and Joëls, 2007).

\section{CLINICAL RELEVANCE \\ BEHAVIORAL CONSEQUENCES OF CHRONIC STRESS}

How could these cellular changes after chronic stress contribute to increased susceptibility to disease and/or the manifestation of psychopathology?

The gradual reduction in 5-HT responsiveness of CA1 hippocampal neurons after chronic stress may contribute to the development of mood disturbances. The morphological changes in the various hippocampal regions have been related to changes in hippocampal function after chronic stress and glucocorticoid exposure (see Conrad, 2006; Tata and Anderson, 2010). Reduced dendritic length is thought to compromise the transfer of signals through the hippocampus and thereby the capacity to learn and memorize information. Reduced neurogenesis could also have a large impact, since young adult-born granule cells contribute significantly to behavioral functions such as contextual fear memory, spatial pattern separation, and spatial learning (Saxe et al., 2006; Zhang et al., 2008; Clelland et al., 2009; Kitamura et al., 2009). Yet, the link between depressive-like behavior and neurogenesis is much disputed (see e.g., DeCarolis and Eisch, 2010). This is corroborated by recent experimental studies showing that development of helplessness (Vollmayr et al., 2003) or anhedonia (Jayatissa et al., 2009) following exposure to inescapable shocks or chronic stress, can occur without impairments in neurogenesis. Conversely, a downregulation in neurogenesis does not necessarily lead to helpless (Santarelli et al., 2003; Vollmayr et al., 2003) or anhedonic-like behaviors (Jayatissa et al., 2009).

Thus, linking behavioral performance to structural plasticity is not entirely straightforward. The behavioral tasks are also often not very specific for a particular subregion of the hippocampus, which makes it difficult to directly relate cell shape or neurogenesis to behavioral performance. Moreover, learning itself may affect spine morphology and neurogenesis, so that 'learning effects' confound effects caused by chronic stress (Leuner et al., 2006; Dupret et al., 2007). It has furthermore been argued that hyperactivity of the HPA-axis at the moment of behavioral testing rather than lasting morphological changes explain differences in memory performance due to chronic stress (Wright et al., 2006). Still, since LTP is greatly hampered in all three hippocampal subareas after chronic stress, deficits in declarative memory are expected to develop along with prolonged stress exposure. In agreement, an overall decline in declarative memory function has been reported in depressed patients (see for example Deuschle et al., 2004).

Clearly, cellular changes after chronic stress - and hence their behavioral consequences - are not restricted to the hippocampus. For instance, the extended dendritic trees of principal cells in the BLA may very well promote anxiety (Roozendaal et al., 2009). In accordance, enhanced anxiety was seen in chronic stress conditions resulting in dendritic changes, whereas conditions that did not affect dendritic structure did not increase anxiety (Vyas and Chattarji, 2004). A second example concerns the structural plasticity seen in the mPFC. Dendritic complexity in the mPFC (but not orbital frontal) was found to correlate with behavioral flexibility (Cerqueira et al., 2005; Dias-Ferreira et al., 2009) and attentional set-shifting performance, i.e., behavioral aspects that depend on mPFC function (Liston et al., 2009). Conversely, reversal learning, which depends on the function of the orbital frontal cortex, was not impaired, which fits with the increased rather than decreased dendritic complexity of pyramidal neurons in the orbitofrontal cortex after chronic stress (Liston et al., 2006).

\section{ANTIDEPRESSANTS AND ANTI-GLUCOCORTICOIDS}

A second set of data also supports the idea that chronic stressinduced structural and functional changes in limbic areas bears relevance to psychopathology. This refers to the observation that treatments with antidepressants often reverse the stress-induced effects. For instance, antidepressant drugs can prevent and/or normalize effects of chronic stress on dendritic remodeling, apoptosis, and increased NMDA-transmission in the CA3 area (Magarinos et al., 1999; Kole et al., 2002; Lucassen et al., 2004). The normalizing effect has been most extensively studied with respect to neurogenesis (Czeh et al., 2001; Santarelli et al., 2003; Drew and Hen, 2007; Pittenger and Duman, 2008; Lucassen et al., 2010a). The observation that different classes of antidepressant drugs, with distinct mechanisms of action, can all block behavioral effects of stress and restore normal levels of adult hippocampal neurogenesis is complemented by experiments showing that neurogenesis is required for the behavioral effects of antidepressants (Santarelli et al., 2003). Together, they support the possibility that stimulating 
neurogenesis is a common pathway through which antidepressant drugs exert their behavioral and therapeutic effects. The link between antidepressant activity and stimulation of neurogenesis, however, is not completely unequivocal. Thus, not all antidepressants stimulate neurogenesis, and both neurogenesis-dependent and neurogenesis-independent mechanisms of antidepressant action exist (Sahay and Hen, 2007; David et al., 2009). Moreover, recent rodent studies have shown that the stimulatory action of the common selective-serotonin re-uptake inhibitor fluoxetine on neurogenesis occurs only in young but not older animals (CouillardDespres et al., 2009) consistent with recent data on the human hippocampus where reductions in proliferation were found in a cohort of elderly depressed cases in the absence of a stimulatory effect of antidepressant treatment (Lucassen et al., 2010b). Another relevant factor in this respect is the clear septo-temporal gradient within the hippocampus in neurogenesis that has been attributed to specific functionalities (Bannerman et al., 2004). Of interest, changes in neurogenesis after stress and antidepressant treatment are generally more pronounced in the ventral hippocampus (Banasr et al., 2006; Sahay and Hen, 2007).

Interestingly, in addition to the well-established antidepressants discussed above, the GR-antagonist RU 38486 also potently protects against chronic stress-induced cellular changes. In humans, this compound was found to quickly (within 1 week) alleviate symptoms of psychotic depression, although very high doses seem to be necessary to exert beneficial effects (DeBattista and Belanoff, 2006). In the rat model of 3 -weeks exposure to unpredictable stress or corticosterone, treatment with RU 38486 only during the last 4 days was already effective in normalizing neurogenesis in the dentate gyrus (Mayer et al., 2006; Oomen et al., 2007), as well as LTP (Krugers et al., 2006) and calcium current amplitude in the CA1 region (Karst and Joëls, 2007). In contrast to antidepressants,

\section{REFERENCES}

Aimone, J. B. Wiles, J., and Gage, F. H. (2006). Potential role for adult neurogenesis in the encoding of time in new memories. Nat. Neurosci. 9, 723-727.

Alfarez,D. N., De Simoni,A., Velzing, E.H., Bracey, E., Joels, M., Edwards, F.A., and Krugers, H. J. (2009). Corticosterone reduces dendritic complexity in developing hippocampal CA1 neurons. Hippocampus 19, 828-836.

Alfarez, D. N., Joëls, M., and Krugers, H. J. (2003). Chronic unpredictable stress impairs long-term potentiation in rat hippocampal CA1 area and dentate gyrus in vitro. Eur. J. Neurosci. 17, 1928-1934.

Alfarez, D. N., Karst, H., Velzing, E. H., Joels, M., and Krugers, H. J. (2008). Opposite effects of glucocorticoid receptor activation on hippocampal CA1 dendritic complexity in chronically stressed and handled animals. Hippocampus 18, 20-28.

Banasr, M., Soumier, A., Hery, M., Mocaer, E., and Daszuta,A. (2006).Agomelatine, a new antidepressant, induces regional

RU 38486 by itself was ineffective when tested in handled control animals. These observations suggest that this drug may derive its beneficial potential in humans from a direct intervention with GR-dependent pathways.

\section{CONCLUDING REMARKS}

We here presented evidence that chronic uncontrollable and unpredictable stress exposure has a great impact on the structure and function of neurons in various limbic regions, particularly in the hippocampus, but also in the BLA and the PFC. There is no reason to assume that chronic stress would not affects other parts of the brain, but so far these areas have been far less investigated.

The available data shows that the impact of chronic stress is not a mere cumulative effect of what is seen after acute stress exposure. If this would be the case, chronic stress would impose a (semi-) permanent condition of what is normally seen several hours after an acute stressor. While this might be true for some parameters (e.g., suppression of LTP, reduction in neurogenesis), it cannot be generalized. For instance, acute GR activation has little effect on glutamatergic transmission in the dentate gyrus, while the same treatment against a background of chronic stress leads to strongly enhanced AMPA-receptor-mediated transmission (Karst and Joëls, 2003). Furthermore, acute stress does not clearly affect dendritic structure of hippocampal pyramidal neurons, yet chronic stress consistently leads to loss of dendritic complexity, particularly in the CA3 region. The consequences of chronic stress for structure and function of limbic cells are therefore complex, though profound. These changes could make individuals more liable to psychopathology. Treatment directed at intervention of these processes alleviates chronic stress-induced alterations and might potentially also ameliorate clinical symptoms in individuals with stress-related psychopathology.

of adult dentate gyrus-derived neural precursor cells through GSK3beta and beta-catenin/TCF pathway. Neuropsychopharmacology 34, 805-815.

Bredy, T. W., Grant, R. J., Champagne, D. L., and Meaney, M. J. (2003). Maternal care influences neuronal survival in the hippocampus of the rat. Eur. J. Neurosci. 18, 2903-2909.

Brown, S. M., Henning, S., and Wellman, C. L. (2005). Mild, short-term stress alters dendritic morphology in rat medial prefrontal cortex. Cereb. Cortex 15, 1714-1722.

Buchanan, T. W., and Lovallo, W. R. (2001). Enhanced memory for emotional material following stresslevel cortisol treatment in humans. Psychoneuroendocrinology 26, 307-317.

Cameron, H. A., Tanapat, P., and Gould, E. (1998). Adrenal steroids and $\mathrm{N}$-methyl-D-aspartate receptor activation regulate neurogenesis in the dentate gyrus of adult rats through a common pathway. Neuroscience 82 349-354.
Caspi, A., Sugden, K., Moffitt, T. E., Taylor, A., Craig, I. W., Harrington, H., McClay, J., Mill, J., Martin, J., Braithwaite, A., and Poulton, R. (2003). Influence of life stress on depression: moderation by a polymorphism in the 5 -HTT gene. Science 301, 386-389.

Castrén, E. (2005). Is mood chemistry? Nat. Rev. Neurosci. 6, 241-246.

Cerqueira, J. J., Pêgo, J. M., Taipa, R., Bessa, J. M., Almeida, O. F., and Sousa, N. (2005). Morphological correlates of corticosteroid-induced changes in prefrontal cortex-dependent behaviors. J. Neurosci. 25, 7792-7800.

Clelland, C. D., Choi, M., Romberg, C., Clemenson, G.D. Jr., Fragniere, A., Tyers, P., Jessberger, S., Saksida, L. M., Barker, R. A., Gage, F. H., and Bussey, T. J. (2009). A functional role for adult hippocampal neurogenesis in spatial pattern separation. Science $325,210-213$.

Conrad, C. D. (2006). What is the functional significance of chronic stressinduced CA3 dendritic retraction within the hippocampus? Behav. Cogn. Neurosci. Rev. 5, 41-60. 
Cook, S. C., and Wellman, C. L. (2004). Chronic stress alters dendritic morphology in rat medial prefrontal cortex. J. Neurobiol. 60, 236-248.

Couillard-Despres, S., Wuertinger, C., Kandasamy, M., Caioni, M., Stadler, K., Aigner, R., Bogdahn, U., and Aigner, L. (2009). Ageing abolishes the effects of fluoxetine on neurogenesis. Mol. Psychiatry 14, 856-864.

Czeh, B., and Lucassen, P. J. (2007). What causes the hippocampal volume decrease in depression? Are neurogenesis, glial changes and apoptosis implicated? Eur. Arch. Gen. Psychiatry Clin. Neurosci. 257, 250-260.

Czeh, B., Michaelis, T., Watanabe, T., Frahm, J., de Biurrun, G., van Kampen, M., Bartolomucci, A., and Fuchs, E. (2001). Stress-induced changes in cerebral metabolites, hippocampal volume, and cell proliferation are prevented by antidepressant treatment with tianeptine. Proc. Natl. Acad. Sci. U.S.A. 98, 12796-12801.

Czéh, B., Simon, M., Schmelting, B., Hiemke, C., and Fuchs, E. (2006). Astroglial plasticity in the hippocampus is affected by chronic psychosocial stress and concomitant fluoxetine treatment. Neuropsychopharmacology 31, 1616-1626.

David, D. J., Samuels, B. A., Rainer, Q., Wang, J. W., Marsteller, D., Mendez, I., Drew, M., Craig, D. A., Guiard, B. P., Guilloux, J. P., Artymyshyn, R. P., Gardier,A.M., Gerald, C., Antonijevic, I. A., Leonardo, E. D., and Hen, R. (2009). Neurogenesis-dependent and -independent effects of fluoxetine in an animal model of anxiety/depression. Neuron 62, 479-493.

DeBattista, C., and Belanoff, J. (2006). The use of mifepristone in the treatment of neuropsychiatric disorders. Trends Endocrinol. Metab. 17, 117-121.

DeCarolis, N. A. and Eisch, A. J. (2010). Hippocampal neurogenesis as a target for the treatment of mental illness: a critical evaluation. Neuropharmacology 58, 884-893.

de Kloet, E. R., Joëls, M., and Holsboer, F. (2005). Stress and the brain: from adaptation to disease. Nat. Rev. Neurosci. 6, 463-475.

Deuschle, M., Kniest, A., Niemann, H., Erb-Bies, M., Colla, N., Hamann, B., and Heuser, I. (2004). Impaired declarative memory in depressed patients is slow to recover: clinical experience. Pharmacopsychiatry 37, 147-151.

Dias-Ferreira, E., Sousa, J. C., Melo, I., Morgado, P., Mesquita, A. R., Cerqueira, J. J., Costa, R. M., and Sousa, N. (2009). Chronic stress causes frontostriatal reorganization and affects decision-making. Science 325, 621-625.
Drew, M. R., and Hen, R. (2007). Adult hippocampal neurogenesis as target for the treatment of depression. CNS Neurol. Disord. Drug Targets 6, 205-218.

Dupret, D., Fabre, A., Döbrössy, M. D., Panatier, A., Rodríguez, J. J., Lamarque, S., Lemaire, V., Oliet, S. H., Piazza, P. V., and Abrous, D. N. (2007). Spatial learning depends on both the addition and removal of new hippocampal neurons. PLoS Biol. 5, e214. doi: 10.1371/ journal.pbio.0050214.

Dupret, D., Revest, J. M., Koehl, M., Ichas, F., De Giorgi, F., Costet, P., Abrous, D. N., and Piazza, P. V. (2008). Spatial relational memory requires hippocampal adult neu- rogenesis. PLoS ONE 3, e1959. doi: 10.1371/journal. pone.0001959.

Duvarci, S., and Paré, D. (2007). Glucocorticoids enhance the excitability of principal basolateral amygdala neurons. J. Neurosci. 27, 4482-4491.

Fenoglio, K.A., Brunson, K. L., and Baram, T.Z. (2006). Hippocampal neuroplasticity induced by early-life stress: functional and molecular aspects. Front. Neuroendocrinol. 27, 180-192.

Galea, L. A., McEwen, B. S., Tanapat, P., Deak, T., Spencer, R. L., and Dhabhar, F. S. (1997). Sex differences in dendritic atrophy of CA3 pyramidal neurons in response to chronic restraint stress. Neuroscience 81, 689-697.

Garthe, A., Behr, J., and Kempermann, G. (2009).Adult-generated hippocampal neurons allow the flexible use of spatially precise learning strategies. PLoS ONE 4, e5464. doi: 10.1371/journal. pone.0005464.

Gould,E. (2007). How widespread is adult neurogenesis in mammals? Nat. Rev. Neurosci. 8, 481-488.

Hains, A. B., Vu, M. A., Maciejewski, P. K., van Dyck, C. H., Gottron, M., and Arnsten, A. F. (2009). Inhibition of protein kinase $\mathrm{C}$ signaling protects prefrontal cortex dendritic spines and cognition from the effects of chronic stress. Proc. Natl. Acad. Sci. U.S.A. 106, 17957-17962.

Heim, C., Newport, D. J., Mletzko, T., Miller, A. H., and Nemeroff, C. B. (2008). The link between childhood trauma and depression: insights from HPA axis studies in humans. Psychoneuroendocrinology 33, 693-710.

Heine, V. M., Maslam, S., Zareno, J., Joëls, M., and Lucassen, P. J. (2004a). Suppressed proliferation and apoptotic changes in the rat dentate gyrus after acute and chronic stress are reversible. Eur. J. Neurosci. 19, 131-144.

Heine, V. M., Maslam, S., Joëls, M., and Lucassen, P. J. (2004b). Increased $\mathrm{P} 27 \mathrm{KIP} 1$ protein expression in the dentate gyrus of chronically stressed rats indicates $\mathrm{G} 1$ arrest involvement. Neuroscience 129, 593-601.

Heine, V. M., Zareno, J., Maslam, S., Joels, M., and Lucassen, P. J. (2005). Chronic stress in the adult dentate gyrus reduces cell proliferation near the vasculature and VEGF and Flk-1 protein expression. Eur. J. Neurosci. 21 , 1304-1314.

Henn, F.A., and Vollmayr, B. (2005). Stress models of depression: forming genetically vulnerable strains. Neurosci. Biobehav. Rev. 29, 799-804.

Holsboer, F., and Ising, M. (2010). Stress hormone regulation: biological role and translation into therapy. Annu. Rev. Psychol. 61, 81-109.

Hu, W., Zhang, M., Czeh. B., Flügge, G. and Zhang, W. (2010). Stress impairs GABAergic network function in the hippocampus by activating nongenomic glucocorticoid receptors and affecting the integrity of the parvalbumin-expressing neuronal network. Neuropsychopharmacology [Epub ahead of print].

Izquierdo, A., Wellman, C. L., and Holmes, A. (2006). Brief uncontrollable stress causes dendritic retraction in infralimbic cortex and resistance to fear extinction in mice. J. Neurosci. 26 5733-5738.

Jayatissa, M. N., Henningsen, K., West, M J., and Wiborg, O. (2009). Decreased cell proliferation in the dentate gyrus does not associate with development of anhedonic-like symptoms in rats. Brain Res. 1290, 133-141.

Jessberger, S., Clark, R. E., Broadbent, N. J., Clemenson, G. D. Jr., Consiglio, A., Lie, D. C., Squire, L. R., and Gage, F. H. (2009). Dentate gyrus-specific knockdown of adult neurogenesis impairs spatial and object recognition memory in adult rats. Learn. Mem. 16, 147-154.

Joëls, M., and Baram, T. Z. (2009). The neuro-symphony of stress. Nat. Rev. Neurosci. 10, 459-466.

Joëls, M., and de Kloet, E. R. (1989). Effects of glucocorticoids and norepinephrine on the excitability in the hippocampus. Science 245, 1502-1505.

Joëls, M., Karst, H., Krugers, H. J., and Lucassen, P. J. (2007). Chronic stress: implications for neuronal morphology, function and neurogenesis. Front. Neuroendocrinol. 28, 72-96.

Karst, H., Berger, S., Turiault, M. Tronche, F., Schütz, G., and Joëls, M. (2005). Mineralocorticoid receptors are indispensable for nongenomic modulation of hippocampal glutamate transmission by corticosterone. Proc. Natl. Acad. Sci. U.S.A. 102, 19204-19207.

Karst, H., and Joëls, M. (2003). Effect of chronic stress on synaptic currents in rat hippocampal dentate gyrus neurons. J. Neurophysiol. 89, 625-633.

Karst, H., and Joëls, M. (2007). Brief RU 38486 treatment normalizes the effects of chronic stress on calcium currents in rat, hippocampal CA1 neurons. Neuropsychopharmacology 32, 1830-1839.

Karst, H., Karten, Y. J., Reichardt, H. M., de Kloet, E. R., Schütz, G., and Joëls, M. (2000). Corticosteroid actions in hippocampus require DNA binding of glucocorticoid receptor homodimers. Nat. Neurosci. 3, 977-978.

Karten, Y. J., Nair, S. M., van Esssen, L., Sibug, R., and Joëls, M. (1999). Longterm exposure to high corticosterone levels attenuates serotonin responses in rat hippocampal CA1 neurons. Proc. Natl. Acad. Sci. U.S.A. 96, 13456-13461.

Kempermann, G., and Kronenberg, G. (2003). Depressed new neuronsadult hippocampal neurogenesis and a cellular plasticity hypothesis of major depression. Biol. Psychiatry 54, 499-503.

Kerr, D. S., Campbell, L. W., Hao, S. Y., and Landfield,P.W. (1989). Corticosteroid modulation of hippocampal potentials: increased effect with aging. Science 245, 1505-1509.

Kim, J. J., and Diamond, D. M. (2002). The stressed hippocampus, synaptic plasticity and lost memories. Nat. Rev. Neurosci. 3, 453-462.

Kitamura, T., Saitoh, Y., Takashima, N., Murayama, A., Niibori, Y., Ageta, H., Sekiguchi, M., Sugiyama, H., and Inokuchi, K. (2009). Adult neurogenesis modulates the hippocampusdependent period of associative fear. Cell 139, 814-827.

Kole, H. P., Swan, L., and Fuchs, E. (2002). The antidepressant tianeptine persistently modulates glutamate receptor currents of the hippocampal CA3 commissural associational synapse tin chronically stressed rat. Eur. J. Neurosci. 16, 807-816.

Kole, M. H., Costoli, T., Koolhaas, J. M., and Fuchs, E. (2004). Bidirectional shift in the cornu ammonis 3 pyramidal dendritic organization following brief stress. Neuroscience 125, 337-347.

Krugers., H. J., Goltstein, P. M., van der Linden, S., and Joëls, M. (2006). Blockade of glucocorticoid receptors rapidly restores hippocampal CA1 synaptic plasticity after exposure to chronic stress. Eur. J. Neurosci. 23, 3051-3055.

Lambert, K. G., Buckelew, S. K., StaffisoSandoz, G., Gaffga, S., Carpenter, W., Fisher, J., and Kinsley, C. H. (1998). Activity-stress induces atrophy of apical dendrites of hippocampal pyramidal neurons in male rats. Physiol. Behav. 65, 43-49. 
Lemaire, V., Koehl, M., Le Moal, M., and Abrous, D. N. (2000). Prenatal stress produces learning deficits associated with an inhibition of neurogenesis in the hippocampus. Proc. Natl. Acad. Sci. U.S.A. 97, 11032-11037.

Leuner, B., and Gould,E. (2010). Structural plasticity and hippocampal function. Annu. Rev. Psychol. 61, 111-140.

Leuner, B., Gould, E., and Shors, T. J. (2006). Is there a link between adult neurogenesis and learning? Hippocampus 16, 216-224.

Liebmann, L., Karst, H., Sidiropoulou, K., van Gemert, N., Meijer, O. C., Poirazi, P., and Joëls, M. (2008). Differential effects of corticosterone on the slow after hyperpolarization in the basolateral amygdala and CA1 region: possible role of calcium channel subunits. J. Neurophysiol. 99, 958-968.

Liston, C., McEwen, B. S., and Casey, B. J. (2009). Psychosocial stress reversibly disrupts prefrontal processing and attentional control. Proc. Natl. Acad. Sci. U.S.A. 106, 912-917.

Liston, C., Miller, M. M., Goldwater, D. S., Radley, J. J., Rocher, A. B., Hof, P. R., Morrison, J. H., and McEwen, B. S. (2006). Stress-induced alterations in prefrontal cortical dendritic morphology predict selective impairments in perceptual attentional set-shifting. $J$. Neurosci. 26, 7870-7874.

Lucassen, P. J., Fuchs, E., and Czéh, B. (2004). Antidepressant treatment with tianeptine reduces apoptosis in the tree shrew hippocampal dentate gyrus and temporal cortex. Biol. Psychiatry 55, 789-796.

Lucassen, P. J., Meerlo, P., Naylor, A. S., van Dam, A. M., Dayer, A. G., Fuchs, E., Oomen, C. A., and Czeh, B. (2010a). Regulation of neurogenesis by stress, sleep, exercise and inflammation; implications for depression and antidepressant action. Eur. Neuropsychopharmacol 20, 1-17.

Lucassen, P. J., Stumpel, M. W., Wang, Q., and Aronica, E. (2010b). Decreased numbers of progenitor cells but no response to antidepressant drugs in the hippocampus of elderly depressed patients. Neuropharmacology 58, 940-949.

Lyons, D. M., Parker, K. J., Zeitzer, J. M., Buckmaster, C. L., and Schatzberg, A. F. (2007). Preliminary evidence that hippocampal volumes in monkeys predict stress levels of adrenocorticotropic hormone. Biol. Psychiatry 62, 1171-1174.

Magarinos, A. M., Deslandes, A., and McEwen, B. S. (1999). Effects of antidepressants and benzodiazepine treatments on the dendritic structure of CA3 pyramidal neurons after chronic stress. Eur. J. Pharmacol. 371, 113-122.
Magarinos, A. M., Li, C. J., Toth, G., Jing, D., Lee, F. S., and McEwen, B. S. (2010). Effect of brain-derived neurotrophic factor haploinsufficiency on stressinduced remodeling of hippocampal neurons. Hippocampus [Epub ahead of print].

Magarinos, A. M., and McEwen, B. S. (1995). Stress-induced atrophy of apical dendrites of hippocampal CA3c neurons: comparison of stressors. Neuroscience 69, 83-88.

Magarinos, A. M., McEwen, B. S., Flugge, G., and Fuchs, E. (1996). Chronic psychosocial stress causes apical dendritic atrophy of hippocampal CA3 pyramidal neurons in subordinate tree shrews. J. Neurosci. 16, 3534-3540.

Magarinos, A. M., Verdugo, J. M., and McEwen, B. S. (1997). Chronic stress alters synaptic terminal structure in hippocampus. Proc. Natl. Acad. Sci. U.S.A. 94, 14002-14008.

Maneschijn, L., van den Akker, E. L. T., Lamberts, S. W. J., and van Rossum, E. F. C. (2009). Clinical features associated with glucocorticoid receptor polymorphisms. Ann. N. Y. Acad. Sci. 1179, 179-198.

Mayer, J. L., Klumpers, L., Maslam, S., de Kloet, E. R., Joëls, M., and Lucassen, P. J. (2006). Brief treatment with the glucocorticoid receptor antagonist mifeprestone normalizes the corticosterone-induced reduction of adult hippocampal neurogenesis. J. Neuroendocrinol. 18, 629-631.

McEwen, B. S. (2000). Effects of adverse experiences for brain structure and function. Biol. Psychiatry 48, 721-731.

McEwen, B. S. (2007). Physiology and neurobiology of stress and adaptation: central role of the brain. Physiol. Rev. 87, 873-904.

McKittrick, C. R., Blanchard, D. C., Blanchard, R. J., McEwen, B. S., and Sakai, R. R. (1995). Serotonin receptor binding in a colony model of chronic social stress. Biol. Psychiatry 37, 383-393.

McKittrick, C. R., Magarinos, A. M., Blanchard, D. C., Blanchard, R. J., McEwen, B. S., and Sakai, R. R. (2000). Chronic social stress reduces dendritic arbors in CA3 of hippocampus and decreases binding to serotonin transporter sites. Synapse 36, 85-94.

Mitra, R., and Sapolsky, R. M. (2008). Acute corticosterone treatment is sufficient to induce anxiety and amygdaloid dendritic hypertrophy. Proc. Natl. Acad. Sci. U.S.A. 105, 5573-5578.

Montaron, M. F., Piazza, P.V., Aurousseau, C., Urani,A., Le Moal, M., and Abrous, D. N. (2003). Implication of corticosteroid receptors in the regulation of hippocampal structural plasticity. Eur. J. Neurosci. 18, 3105-3111.

Munafo, M. R., Durrant, C., Lewis, G., and Flint, J. (2009). Gene x environment interactions at the serotonin transporter locus. Biol. Psychiatry 65 , 211-219.

Nacher, J., and McEwen, B. S. (2006). The role of $\mathrm{N}$-methyl-D-asparate receptors in neurogenesis. Hippocampus $16,267-270$.

Olijslagers, J. E., de Kloet, E. R., Elgersma, Y., van Woerden, G. M., Joëls, M., and Karst, H. (2008). Rapid changes in hippocampal CA1 pyramidal cell function via pre- as well as postsynaptic membrane mineralocorticoid receptors. Eur. J. Neurosci. 27, 2542-2550.

Oomen, C. A., Girardi, C. E., Cahyadi, R., Verbeek, E. C., Krugers, H. J., Joëls, M., and Lucassen, P. J. (2009). Opposite effects of early maternal deprivation on neurogenesis in male versus female rats. PLoS ONE 3, e3675. doi: 10.1371/ journal.pone.0003675.

Oomen, C. A., Mayer, J. L., de Kloet, E. R., Joëls, M., and Lucassen, P. J. (2007). Brief treatment with the glucocorticoid receptor antagonist mifepristone normalizes the reduction in neurogenesis after chronic stress. Eur. J. Neurosci. 26, 3395-3401.

Oomen, C. A., Soeters, H., Audureau, N., Vermunt, L., van Hasselt, F., Joëls, M., Lucassen, P. J., and Krugers, H. J. (2010). Reduced neurogenesis and altered cellular morphology after early maternal deprivation is paralleled by impaired water maze aquisition but increases in fear memory in the adult male rat. J. Neurosci.30, 6635-6645.

Pavlides, C., Nivon, L. G., and McEwen, B. S. (2002). Effects of chronic stress on hippocampal long-term potentiation. Hippocampus 12, 245-257.

Pawlak, R., Shankaranarayana Rao, B. S., Melchor, J. P., Chattarji, S., McEwen, B. S., and Strickland, S. (2005). Tissue plasminogen activator and plasminogen mediate stress-induced decline of neuronal and cognitive functions in the mouse hippocampus. Proc. Natl. Acad. Sci. U.S.A. 102, 18201-18206.

Pham, K., Nacher, J., Hof, P. R., and McEwen, B. S. (2003). Repeated restraint stress suppresses neurogenesis and induces biphasic PSA-NCAM expression in the adult rat dentate gyrus. Eur. J. Neurosci. 17, 879-886.

Pittenger, C., and Duman, R. S. (2008). Stress, depression, and neuroplasticity: a convergence of mechanisms. Neuropsychopharmacology 33, 88-109.

Radley, J. J., Rocher, A. B., Miller, M., Janssen, W. G., Liston, C., Hof, P. R., McEwen, B. S., and Morrison, J. H. (2006). Repeated stress induces dendritic spine loss in the rat medial prefrontal cortex. Cereb. Cortex. 16, 313-320.

Reif, A., Fritzen, S., Finger, M., Strobel, A., Lauer, M., Schmitt, A., and Lesch, K. P. (2006). Neural stem cell proliferation is decreased in schizophrenia, but not in depression. Mol. Psychiatry 11, 514-522.

Revest, J.M., Dupret, D., Koehl, M., FunkReiter, C., Grosjean, N., Piazza, P. V., and Abrous, D. N. (2009). Adult hippocampal neurogenesis is involved in anxiety-related behaviors. Mol. Psychiatry 14, 959-967.

Roozendaal, B., McEwen, B. S., and Chattarji, S. (2009). Stress, memory and the amygdala. Nat. Rev. Neurosci. $10,423-433$.

Sahay, A., and Hen, R. (2007). Adult hippocampal neurogenesis in depression. Nat. Neurosci. 10, 1110-1115.

Sandi, C., Davies, H. A., Cordero, M. I., Rodriguez, J. J., Popov, V. I., and Stewart, M. G. (2003). Rapid reversal of stress induced loss of synapses in CA3 of rat hippocampus following water maze training. Eur. J. Neurosci. 17, 2447-2456.

Santarelli, L., Saxe, M., Gross, C., Surget, A., Battaglia, F., Dulawa, S., Weisstaub, N., Lee, J., Duman, R., Arancio, O., Belzung, C., and Hen, R. (2003). Requirement of hippocampal neurogenesis for the behavioral effects of antidepressants. Science 301, 805-809.

Sapolsky, R. M. (2000). Glucocorticoids and hippocampal atrophy in neuropsychiatric disorders. Arch. Gen. Psychiatry 57, 925-935.

Saxe, M. D., Battaglia, F., Wang, J. W., Malleret, G., David, D. J., Monckton, J. E., Garcia, A. D., Sofroniew, M. V., Kandel, E. R., Santarelli, L., Hen, R., and Drew, M. R. (2006). Ablation of hippocampal neurogenesis impairs contextual fear conditioning and synaptic plasticity in the dentate gyrus. Proc. Natl. Acad. Sci. U.S.A. 103, 17501-17506.

Schloesser, R. J., Manji, H. K., and Martinowich, K. (2009).Suppression of adult neurogenesis leads to an increased hypothalamo-pituitaryadrenal axis response. Neuroreport 20 , 553-557.

Seib, L. M., and Wellman, C. L. (2003). Daily injections alter spine density in rat medial prefrontal cortex. Neurosci. Lett. 337, 29-32.

Sheline, Y. I., Gado, M. H., and Kraemer, H. C. (2003). Untreated depression and hippocampal volume loss. Am. J. Psychiatry 160, 1516-1518.

Smeets, T., Wolf, O. T., Giesbrecht, T., Sijstermans, K., Telgen, S., and Joëls, M. (2009). Stress selectively and lastingly promotes learning of contextrelated high arousing information. 
Psychoneuroendocrinology 34 , 1152-1161.

Sousa, N., Lukoyanov, N. V., Madeira, M. D., Almeida, O. F., and Paula-Barbosa, M. M. (2000). Reorganization of the morphology of hippocampal neurites and synapses after stress-induced damage correlates with behavioral improvement. Neuroscience 97, 253-266.

Stewart, M. G., Davies, H. A., Sandi, C., Kraev, I.V., Rogachevsky, V.V., Peddie, C. J., Rodriguez, J. J., Cordero, M. I., Donohue, H. S., Gabbott P. L. A., and Popov, I. V. (2005). Stress suppresses and learning induces plasticity in CA3 of rat hippocampus: a three-dimensional ultrastructural study of thorny excrescences and their postsynaptic densities. Neuroscience 131, 43-54.

Tata, D. A., and Anderson, B. J. (2010). The effects of chronic glucocorticoid exposure on dendritic length, synapse numbers and glial volume in animal models: implications for hippocampal volume reductions in depression. Physiol. Behav. 99, 186-193.

Tata, D.A., Marciano, V.A., and Anderson, B. J. (2006). Synapse loss from chronically elevated glucocorticoids: relationship to neuropil volume and cell number in hippocampal area CA3. J. Comp. Neurol. 498, 363-374.

Ulrich-Lai, Y. M., and Herman, J. P. (2009). Neural regulation of endocrine and autonomic responses. Nat. Rev. Neurosci. 10, 397-409.

Van Gemert, N., and Joëls, M. (2006). Effect of chronic stress and mifepris- tone treatment on voltage-dependent $\mathrm{Ca} 2+$ currents in rat hippocampal dentate gyrus. J. Neuroendocrinol. 18, 732-741.

Van Gemert, N. G., Carvalho, D. M., Karst, H., van der Laan, S., Zhang, M., Meijer, O. C., Hell, J. W., and Joëls, M. (2009). Dissociation between rat hippocampal CA1 and dentate gyrus cells in their response to corticosterone: effects on calcium channel protein and current. Endocrinology 150, 4615-4624.

Van Riel, E., Meijer, O. C., Steenbergen, P. J., and Joëls, M. (2003). Chronic unpredictable stress causes attenuation of serotonin responses in cornu ammonis 1 pyramidal neurons. Neuroscience 120, 649-658.

Van Rossum, E. F., Binder, E. B., Majer, M., Koper, J. W., Ising, M., Modell, S., Salyakina, D., Lamberts, S. W., and Holsboer, F. (2006). Polymorphisms of the glucocorticoid receptor gene and major depression. Biol. Psychiatry 59, 681-688.

Vollmann-Honsdorf, K., Flugge, G., and Fuchs, E. (1997). Chronic psychosocial stress does not affect the number of pyramidal neurons in tree shrew hippocampus. Neurosci. Lett. 233, 121-124.

Vollmayr B, Simonis C, Weber S, Gass P, and Henn F. (2003). Reduced cell proliferation in the dentate gyrus is not correlated with the development of learned helplessness. Biol. Psychiatry 54, 1035-1040.

Vyas, A., and Chattarji, S. (2004). Modulation of different states of anxiety-like behavior by chronic stress. Behav. Neurosci. 118 , 1450-1454.

Vyas, A., Jadhav, S., and Chattarji, S. (2006). Prolonged behavioral stress enhances synaptic connectivity in the basolateral amygdala. Neuroscience $143,387-393$.

Vyas,A., Mitra, R., Shankaranarayana Rao, B. S., and Chattarji, S. (2002). Chronic stress induces contrasting patterns of dendritic remodeling in hippocampal and amygdaloid neurons. J. Neurosci. 22, 6810-6818.

Watanabe, Y., Gould, E., Cameron, H. A., Daniels, D. C., and McEwen, B. S. (1992). Phenytoin prevents stress- and corticosterone-induced atrophy of CA3 pyramidal neurons. Hippocampus 2, 431-435.

Wellman, C. L. (2001). Dendritic reorganization in pyramidal neurons in medial prefrontal cortex after chronic corticosterone administration. J. Neurobiol. 49, 245-253.

Wiegert, O., Joëls, M., and Krugers, H. (2006). Timing is essential for rapid effects of corticosterone on synaptic potentiation in the mouse hippocampus. Learn. Mem. 13, 110-113.

Woolley, C. S., Gould, E., and McEwen, B. S. (1990). Exposure to excess glucocorticoids alters dendritic morphology of adult hippocampal pyramidal neurons. Brain Res. 31, 225-231.

Wright, R. L., Lightner, E. N., Harman, J. S., Meijer, O. C., and Conrad, C. D. (2006).Attenuating corticosterone lev- els on the day of memory assessment prevents chronic stress-induced impairments in spatial memory. Eur. J. Neurosci. 24, 595-605.

Zhang, C. L., Zou, Y., He, W., Gage, F. H., and Evans, R. M. (2008). A role for adult TLX-positive neural stem cells in learning and behaviour. Nature 451, 1004-1007.

Zhao, C., Deng, W., and Gage, F. H. (2008). Mechanisms and functional implications of adult neurogenesis. Cell 132, 645-660.

Conflict of Interest Statement: The authors declare that the research was conducted in the absence of any commercial or financial relationships that could be construed as a potential conflict of interest.

Received: 07 April 2010; paper pending published: 03 May 2010; accepted: 07 June 2010; published online: 12 July 2010.

Citation: Krugers HJ, Lucassen PJ, Karst H and JoëlsM (2010) Chronic stress effects on hippocampal structure and synaptic function: relevance for depression and normalization by anti-glucocorticoid treatment. Front. Syn. Neurosci. 2:24. doi: 10.3389/ fnsyn.2010.00024

Copyright $\odot 2010$ Krugers, Lucassen, Karst and Joëls. This is an open-access article subject to an exclusive license agreement between the authors and the Frontiers Research Foundation, which permits unrestricted use, distribution, and reproduction in any medium, provided the original authors and source are credited. 